\title{
The dielectric and electrical proprieties of Ethylcarbazole family based copolymer
}

\author{
Hussam Bouaamlat ${ }^{1, *}$, Nasr Hadi ${ }^{2}$, Hayat Sadki ${ }^{3}$, Mohammed Naciri Bennani ${ }^{3}$, Farid Abdi ${ }^{2}$, Tajeddine \\ Lamcharfi $^{2}$, Mohammed Bouachrine ${ }^{4}$ and Mustapha Abarkan ${ }^{1}$ \\ ${ }^{1}$ LSI, Laboratory of Engineering Sciences, Polydisciplinary Faculty of Taza, Sidi Mohamed Ben Abdellah \\ University of Fez, B.P. 1223, Taza, Morocco \\ ${ }^{2}$ LSSC Department of Electrical Engineering, FST, Road Immouzer, Fez - 2202, Morocco \\ ${ }^{3}$ Laboratory of Chemistry-Biology Applied to the Environment, Research team " Applied Materials and \\ Catalyses " Chemistry Department, Faculty of Sciences, Moulay-Ismail University, \\ BP 11201 Zitoune Meknes, Morocco \\ ${ }^{4}$ Natural Substances and Molecular Chemistry Laboratory, Moulay-Ismail University, Morocco
}

\begin{abstract}
The dielectric and electrical proprieties of Ethylcarbazole family based copolymer such as Poly(Ethylcarbazole-Terphenyl) (PEcbz-Ter), Poly(Ethylcarbazole-Anthracene) (PEcbz-Ant) and Poly(Ethylcarbazole-Furan) (PEcbz-Fur) were investigated over the frequency range from $1 \mathrm{kHz}$ to $2 \mathrm{MHz}$. The dielectric constant $\varepsilon_{\mathrm{r}}^{\prime}$ for (PEcbz-Ter), (PEcbz-Ant) and (PEcbz-Fur) pellets was found to decrease as the frequency increases and it was recorded a value of $11 \sim 7,11 \sim 6$ and $7 \sim 6$ respectively at $1 \mathrm{kHz}$. The dielectric loss was investigated and have a value below $1 \%$ at $11 \mathrm{kHz}$, which is suitable for several energy storage applications.
\end{abstract}

Keywords: Conducting polymer; Ethylcarbazole based copolymer; Dielectric proprietie.

\section{Introduction}

Conjugated polymers materials are pervasive in modern electronics and electrical systems. Conducting polymers made of conjugated polymers have found applications in the areas of capacitive energy storage ${ }^{1,2}$, transistors ${ }^{3,4}$, photovoltaic devices 5,6 and electrical insuation ${ }^{7}$. They gained scholars attention due to their optical and electrical properties such as dielectric constant, refractive index, impedance, etc ${ }^{8}$. These parameters carry beneficial information for optimizations of studied materials in technological applications. Indeed, they have the advantage of possessing optical and electronic properties that can be modulated by molecular and supramolecular engineering. Among all those polymers, Carbazole, has been widely used as building blocks in the field of conjugated polymers for electronic applications ${ }^{9,10}$. Carbazole it is a fully aromatic system, very stable to environmental and chemical degradation. Furthermore, its nitrogen group can be easily substituted to provide solubility to the polymer ${ }^{11}$. The carbazole based compounds exhibit high thermal and photochemical stability and some unique electrical properties ${ }^{12}$.
Polymers have been widely used as dielectric materials due to their ease of processing, permitting them to be formed into flexible films ${ }^{13}$. On other hand, high dielectric constant and low dielectric loss polymers are highly desired for advanced electrical applications, such as film capacitors ${ }^{14}$, artificial muscle ${ }^{15}$, and electrocaloric cooling ${ }^{16}$. However, there is no detail studies about dielectric proprieties of carbazole based copolymers such as Ethylcarbazole Poly(Ethylcarbazole-Terphenyl) (PEcbz-Ter), Poly(Ethylcarbazole-Anthracene) (PEcbz-Ant) and Poly(Ethylcarbazole-Furan) (PEcbz-Fur).

In this paper we report at first a characterization measurement based on X-ray diffraction to obtain an insight into the structure of a family of materials based on Ethylcarbazole, PEcbz-Ter, PEcbz-Ant and PEcbz-Fur. The dielectric constant, dielectric loss and the AC conductivity mechanism of these copolymers as function of frequency and temperature range from $1 \mathrm{kHz}$ to $2 \mathrm{MHz}$ and room temperature to $120{ }^{\circ} \mathrm{C}$ respectively are measured and compared. 


\section{Materials and methods}

\section{Preparation of copolymers}

Poly(9-Ethylcarbazole) (PEcbz) powder (97\%), p-Terphenyl (Ter) (99\%), Anthracene (Ant) (99\%) and Furan (Fur) (99\%), ferrichloride (FeCl3), chloroform $\mathrm{CHCl} 3$ (99\%), methanol $\mathrm{CHOH}(99,9 \%)$, used for the synthesis of the studied compounds were purchased from Aldrich chemistry, A Fisher Scientific International and Riedel-de Haën and were purified before use by usual procedures. In this work, the copolymers PEcbz-Ter, PEcbz-Ant and PEcbzFur are dissolved in Chloroform (HPLC grade) and oxidatively polymerized with $\mathrm{FeCl}_{3}{ }^{17,18}$, Furan have been prepared the same way as the previous copolymers.

\section{Dielectric measurements}

Dielectric characterization was carried out on a grounded powder of the Ethylcarbazole based materials which was pressed by a hydraulic press under $\sim 50 \mathrm{MPa}$ to form a pellet with a disk shape. The surfaces of the pellets are well-polished to dryness and metallized by a thin layer of silver paste in order to obtain two parallel plates; the pellets had the same radius as the electrodes which are $12 \mathrm{~mm}$ in a circular disk shape and the thickness were between $1.75-1.80 \mathrm{~mm}$. The real $\left(\varepsilon_{\mathrm{r}}^{\prime}\right)$ part of the dielectric constant of the material was calculated by using the formula:

$$
\varepsilon_{\mathrm{r}}^{\prime}=\mathrm{C}_{\mathrm{p}} \mathrm{d} / \mathrm{A} \varepsilon_{0}
$$

where $C_{p}$ is the capacitance of the sample, $d$ is the thickness of the disk, $\mathrm{A}$ is the surface area of the electrode, $\varepsilon_{0}=8.85 \times 10^{-12} \mathrm{~F} / \mathrm{m}$ the permittivity of free space. The capacitance $\left(\mathrm{C}_{\mathrm{p}}\right)$ and loss factor $(\tan (\delta)$ or $\mathrm{D})$ can be obtained directly from the measurements. The amplitude of the ac electric signal applied to the samples was $1 \mathrm{~V}$.

\section{X-ray diffraction analysis}

XRD measurements were taken to examine the structure of the copolymers. The microanalysis measurements were carried out with X-ray diffraction studies on an Philips PW1800 diffractometer equipped with a curved detector $(\mathrm{K} \alpha$ ray of copper $\lambda=1,5418 \AA$ ).

\section{Results and Discussion}

\section{X-ray diffraction}

$\mathrm{X}$-ray diffraction was made on the materials that were prepared as a powder and the scanning conditions were the same for all of the materials. Figure 2 shows the XRD patterns of the obtained copolymers PEcbz-Ter, PEcbz-Ant and PEcbz-Fur prepared by $\mathrm{FeCl} 3$ coupling reaction. Five and six major peaks are observed at $2 \theta$ equal to $6^{\circ}, 9.5^{\circ}, 10^{\circ}$, $11.5^{\circ}, 14.5^{\circ}$ and $9.6^{\circ}, 19.5^{\circ}, 21^{\circ}, 25^{\circ}, 26^{\circ}, 29^{\circ}$ for PEcbz-Ter and PEcbz-Ant respectively, these peaks are intensified a marked way. The reflection of low intensity is observed in the region of high angle $\left(15^{\circ}-20^{\circ}\right)$ for PEcbz-Ter and $\left(30^{\circ}-35^{\circ}\right)$ for PEcbzAnt, which reflects a new morphology of the copolymer. The most intense peaks at $9.5^{\circ}$ and $19.5^{\circ}$ for PEcbz-Ter and PEcbz-Ant respectively reflect a structural order in our copolymers (crystalline regions). This has been also checked by a scanning electron microscopy (SEM) [17]. However, for the copolymer PEcbz-Fur a very weak and broad diffraction peaks appear around $2 \theta$ equal to $12^{\circ}$ and $22^{\circ}$ (Fig.1c), This may be attributed to a partially ordered structure for this copolymer.

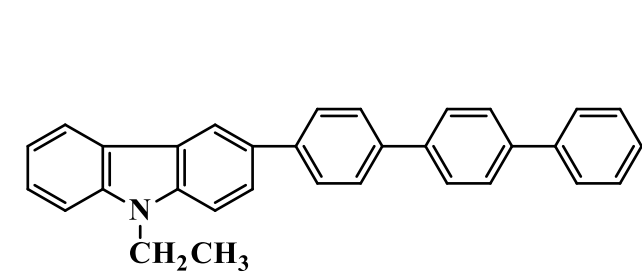

Poly(Ethylcarbazole-Terphenyl)<smiles>CCn1c2ccccc2c2cc(-c3c4ccccc4cc4ccccc34)ccc21</smiles>

Poly(Ethylcarbazole-Anthracene)<smiles>CCn1c2ccccc2c2cc(-c3ccco3)ccc21</smiles>

Poly(Ethylcarbazole-Furanl)

Figure 1. Structures of the studied materials. 

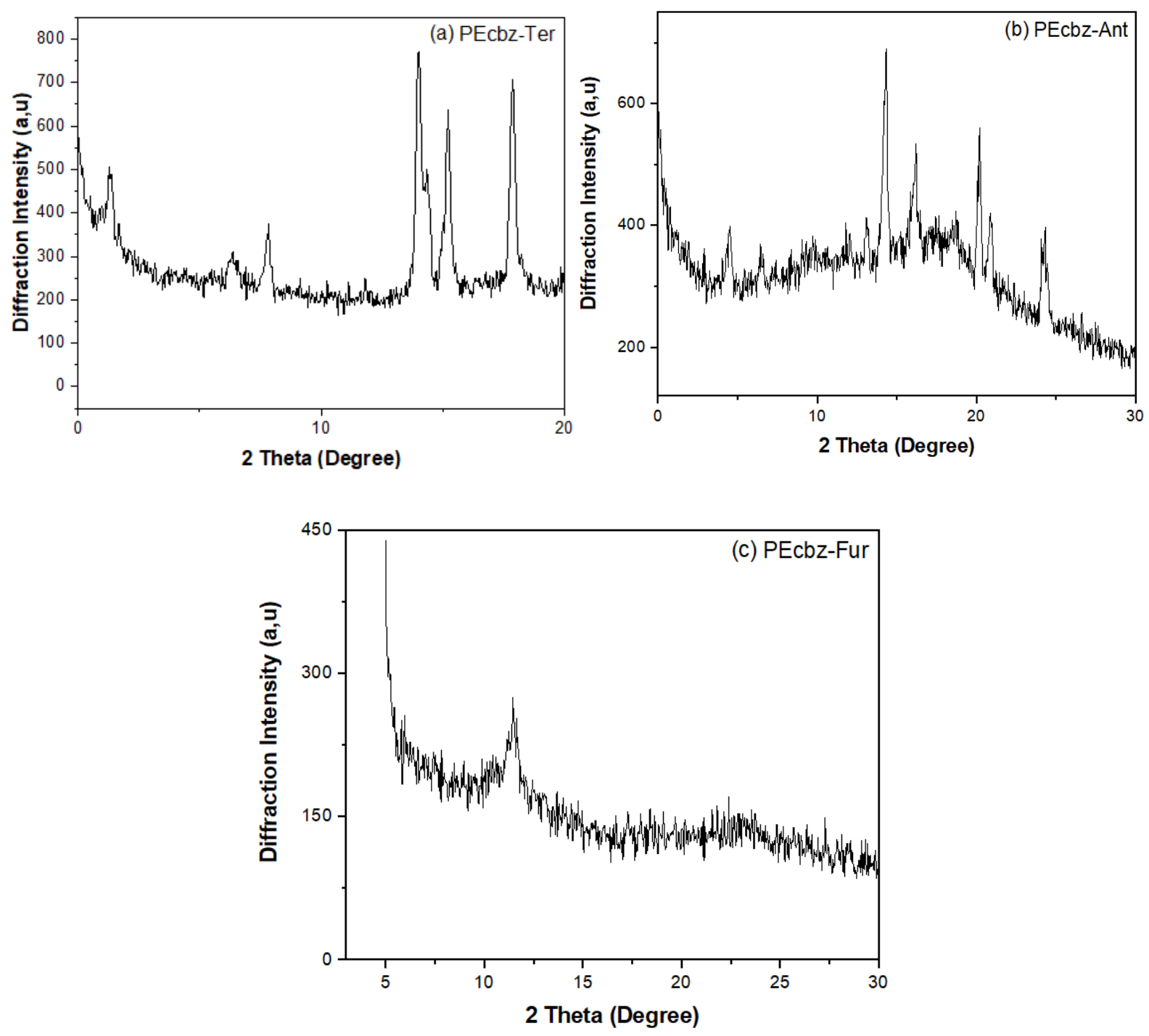

Figure 2. XRD pattern spectra of a) PEcbz-Ter, b) PEcbz-Ant and c) PEcbz-Fur.

\section{Dielectric proprieties}

The results for dielectric constant $\left(\varepsilon_{\mathrm{r}}^{\prime}\right)$ for the PEcbz-Ter, PEcbz-Ant and PEcbz-Fur against frequency at select few temperatures are plotted in Figure 3. For all samples, at low frequency, dielectric constant rapidly decreases then exhibit a step at higher frequencies. This decrease may be due to the incapability of the dipoles to rotate fastly enough so they oscillate behind the field and it's called dielectric relaxation. A phenomenon that tells that the charge carrier localization is not stable and affected by the frequency disturbances ${ }^{19}$. As it observed from the Figure.3, the dielectric constant increases with increasing temperature for the PEcbzTer, PEcbz-Ant samples (Fig.3a and b), while the $\varepsilon_{\mathrm{r}}^{\prime}$ values of PEcbz-Fur decrease with temperature (Fig.3c). The increase observed in $\varepsilon_{\mathrm{r}}^{\prime}$ values with temperature can be explained on the basis that the contribution of the charge carriers to the polarization is weak if they are not in the direction of the field and this affects the change of $\varepsilon_{\mathrm{r}}^{\prime}$ with temperature. Thus, the increase in temperature results in sufficient thermal excitation energy obtained by the bound charge carriers, which enhances the polarization leading to the increase in the dielectric constant ${ }^{20}$. In order to compare the behaviour of the dielectric constant for the three materials at R.T $=30{ }^{\circ} \mathrm{C}$ and $120{ }^{\circ} \mathrm{C}$ at various frequencies we presented them in Table 1. From the Table it is observed that the dielectric constant of PEcbz-Ter has higher values when compared with PEcbz-Fur and PEcbz-Ant. These results showed that changing the substitute lead to a variation in dielectric constant, whereas replace the Furan part with Terphenyl, brought the dielectric constant up to 11 , which is similar to the value for pure $\mathrm{p}$ (DMTSub), These values can be considered high compared with many other organic compounds ${ }^{21}$. 

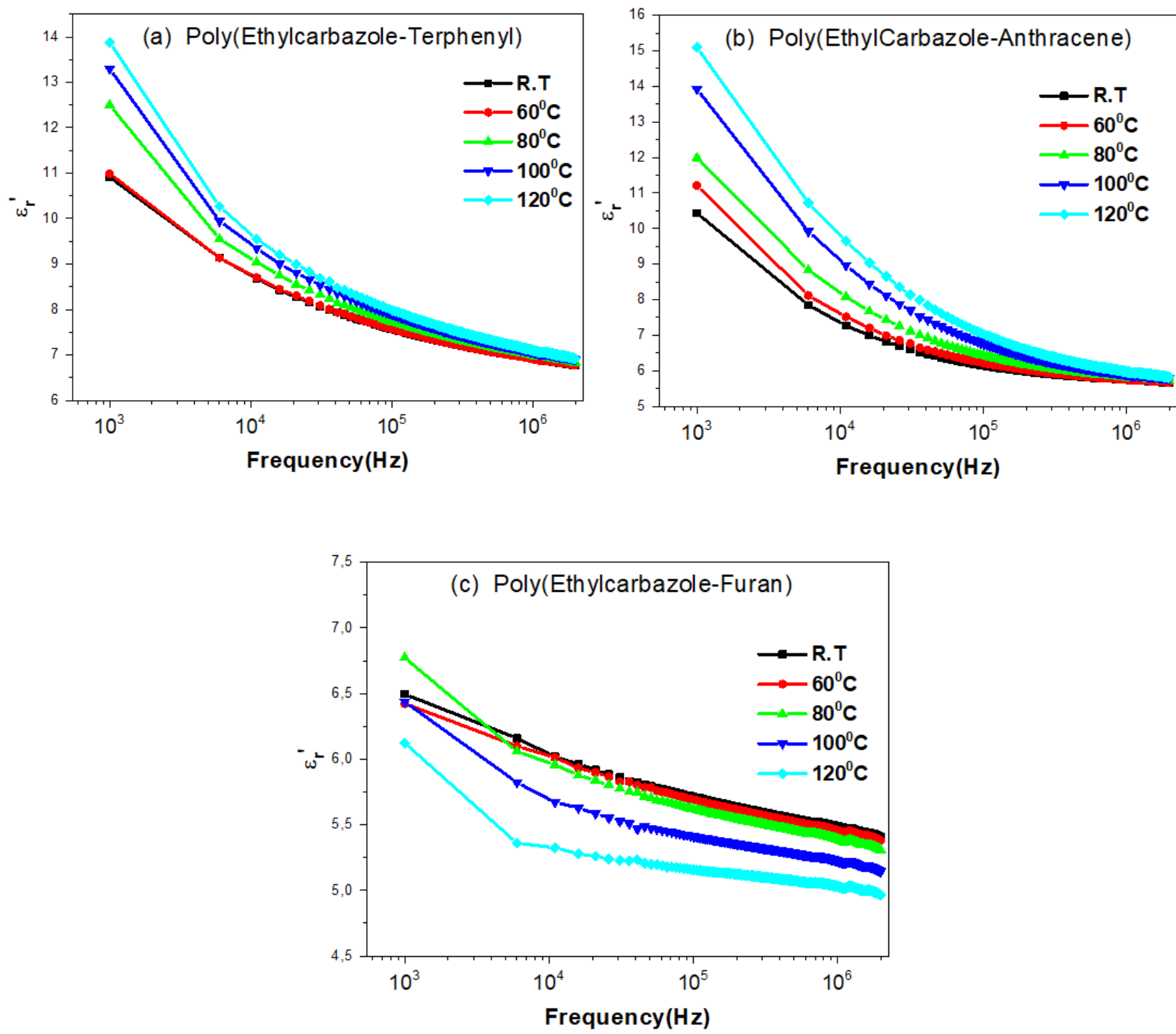

Figure 3. Dielectric constant of a) PEcbz-Ter, b) PEcbz-Ant and c) PEcbz-Fur as a function of frequency at different temperatures.

Furthermore, loss factor results for PEcbz-Ter, PEcbz-Ant and PEcbz-Fur at $11 \mathrm{kHz}, 600 \mathrm{kHz}$ and $2000 \mathrm{kHz}$ are listed in Table 1 at R.T and $120{ }^{\circ} \mathrm{C}$. It is clear from the data presented in the table that for all compounds factor loss obtained increases with increasing temperature and decreases with increasing frequency, on the other hand at higher frequencies the factor loss becomes less sensitive to both temperature and frequency. The behaviour of increasing at low frequency of the loss factor is due to dipole polarization. As far as frequency is increasing, the dipolar polarization effect will tend to zero, then the dielectric loss factor tends to be very low, as shown in Table 1. The values of loss factor of all compounds are below $1 \%$ which make them useful and acceptable for many energy storage applications.

Table 1. Dielectric Constant $\left(\varepsilon_{\mathrm{r}}^{\prime}\right)$ and Dielectric Loss $(\tan \delta)$ at selected frequency.

\begin{tabular}{|c|c|c|c|c|c|}
\hline \multirow[b]{2}{*}{ Compound } & \multirow[b]{2}{*}{ Frequency $(\mathbf{k H z})$} & \multicolumn{2}{|c|}{ AT R.T } & \multicolumn{2}{|c|}{$\operatorname{AT} 120^{\circ} \mathrm{C}$} \\
\hline & & $\varepsilon_{\mathrm{r}}^{\prime}$ & $\tan \delta$ & $\varepsilon_{\mathrm{r}}^{\prime}$ & $\tan \delta$ \\
\hline \multirow[t]{3}{*}{ PEcbz-Ter } & 11 & 8.67 & 0.15 & 9.54 & 0.23 \\
\hline & 600 & 7.03 & 0.05 & 7.26 & 0.07 \\
\hline & 2000 & 6.76 & 0.04 & 6.91 & 0.05 \\
\hline \multirow[t]{3}{*}{ PEcbz-Ant } & 11 & 7.26 & 0.24 & 9.65 & 5.59 \\
\hline & 600 & 13.31 & 0.04 & 6.12 & 0.10 \\
\hline & 2000 & 5.65 & 0.02 & 5.81 & 0.06 \\
\hline \multirow[t]{3}{*}{ PEcbz-Fur } & 11 & 6.01 & 0.05 & 5.32 & 0.03 \\
\hline & 600 & 5.53 & 0.02 & 5.06 & 0.06 \\
\hline & 2000 & 5.40 & 0.02 & 4.97 & 0.09 \\
\hline
\end{tabular}



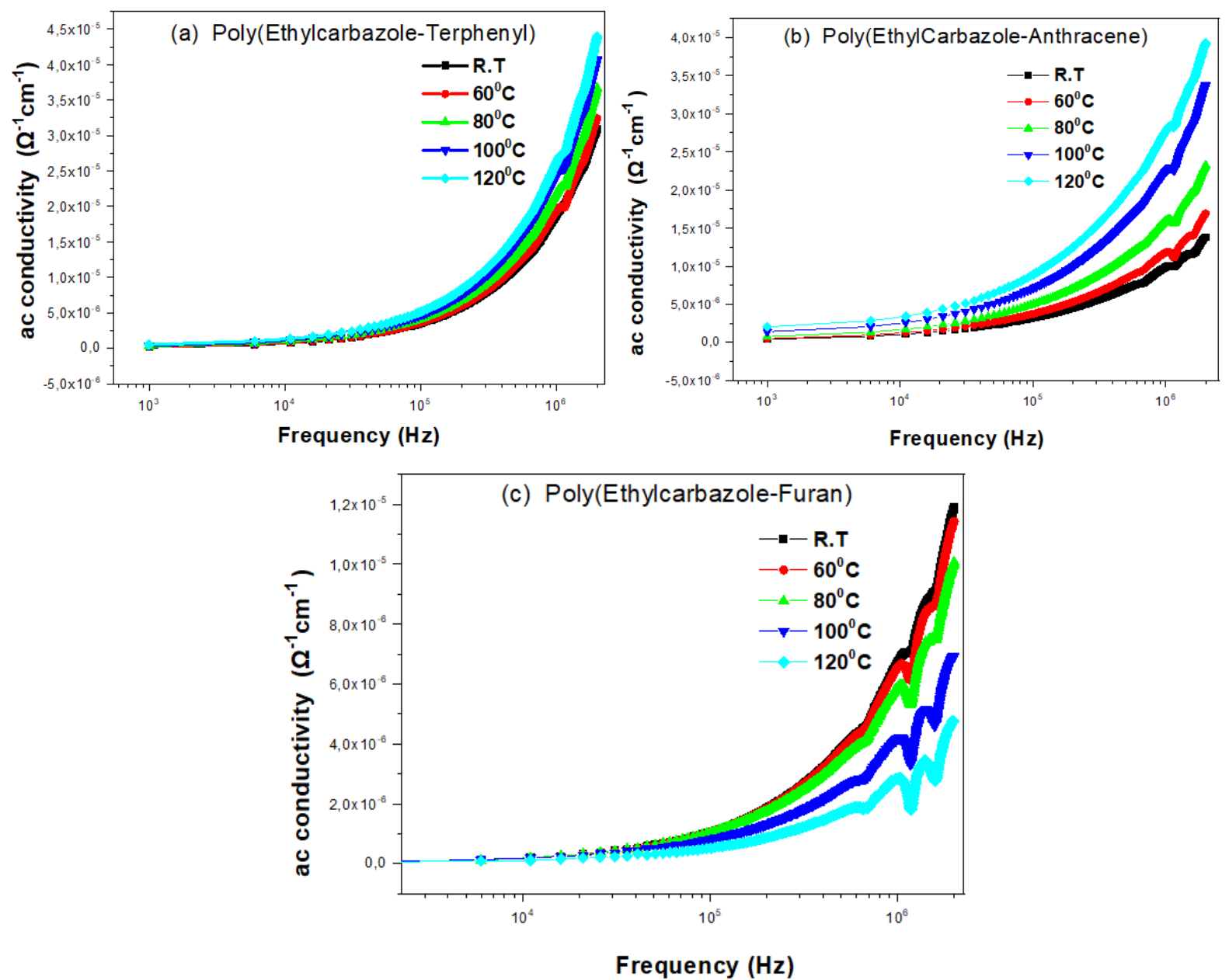

Figure 4. Ac conductivity ( $\sigma_{\mathrm{ac}}$ ) of a) PEcbz-Ter, b) PEcbz-Ant and c) PEcbz-Fur as a function of frequency at different temperatures.

Figure 4 shows the ac conductivity $\sigma_{\mathrm{ac}}$ as a function of frequency at different temperatures of the copolymers. It is found for all compounds that $\sigma_{\mathrm{ac}}$ at the studied temperatures slowly increases with an increase in the frequency, especially in the range up to $10^{5} \mathrm{~Hz}$. After that a steep slope is observed at higher frequencies, which reveals that the $\sigma_{\mathrm{ac}}$ is proportional to mobility and carrier concentrations. The low value of electrical conductivity is the effect of decrease in mobility of the charge carriers due to ionic size, which leads to the change in electronic band structure. The increase in the AC conductivity at high frequencies could be due to the hopping mechanism. A model that was originally proposed by Pike ${ }^{22}$ in order to calculate the frequency dependent conductivity in scandium oxide films, and then extended by Elliott ${ }^{23}$, in the study of chalcogenide glasses. This model suggests a random distribution of the localized states and the motion of electronic charge carriers through a rigorous relaxation time between a pair consisting of two localized states. After changing the furan with the Terphenyl and Anthracene the conductivity was found to change from $10^{-6}$ to $10^{-5} \mathrm{~S} / \mathrm{cm}$, at high frequencies. Since the conductivity seems to depend upon the linkage in the backbone bonding and since phenyl-phenyl bond exhibits greater orbital overlap between phenyl groups, this linkage may well be the structural parameter responsible for the slight differences in conductivity.

Table 2. Ac conductivity $\sigma_{\mathrm{ac}}$ as function of frequency at selected temperatures.

\begin{tabular}{|c|c|c|c|c|c|c|}
\hline & \multicolumn{3}{|c|}{$\sigma_{\mathrm{ac}} / \mathbf{\Omega}^{-1} \mathbf{c m}^{-1}$ At R.T } & \multicolumn{3}{|c|}{$\sigma_{\mathrm{ac}} / \mathbf{\Omega}^{-1} \mathrm{~cm}^{-1} \mathrm{AT} 120^{\circ} \mathrm{C}$} \\
\hline Compound & $11 \mathrm{kHz}$ & $600 \mathrm{kHz}$ & $2000 \mathrm{kHz}$ & $11 \mathrm{kHz}$ & $600 \mathrm{kHz}$ & $2000 \mathrm{kHz}$ \\
\hline PEcbz-Ter & $7,935 * 10^{-7}$ & $1,2861 * 10^{-5}$ & $3,095 * 10^{-5}$ & $1,359 * 10^{-6}$ & $1,803 * 10^{-5}$ & $4,399 * 10^{-5}$ \\
\hline PEcbz-Fur & $2,009 * 10^{-7}$ & $4,337 * 10^{-6}$ & $1,188 * 10^{-5}$ & $1,173 * 10^{-7}$ & $1,878 * 10^{-6}$ & $4,750 * 10^{-6}$ \\
\hline PEcbz-Ant & $1,492 * 10^{-7}$ & $1,308 * 10^{-6}$ & $2,446 * 10^{-6}$ & $3,420 * 10^{-6}$ & $2,150 * 10^{-5}$ & $3,930 * 10^{-5}$ \\
\hline
\end{tabular}


The effect of temperature on the AC conductivity at several frequencies for all copolymers were studied. Figure 5a represents the results of PEcbz-Ter sample as an example. The figure shows the conductivity plot as function of $1000 / \mathrm{T}$ in the temperature range from R.T to $120^{\circ} \mathrm{C}$ at $1 \mathrm{kHz}, 10 \mathrm{kHz}, 50 \mathrm{kHz}$ and $100 \mathrm{kHz}$. From the figure it can be observed that the conductivity slightly changes till $\sim 50{ }^{\circ} \mathrm{C}$ then increases with increase in temperature. This increase at high temperature could be elucidated on the basis that temperature affects the hydrogen bond strength in the molecules, high temperatures lead to the movements of thermally excited carriers from energy levels within the band gap. While the slight changes observed at low temperatures are dominated by the hopping of carriers between localized states. As it observed from the figure that the conductivity has a

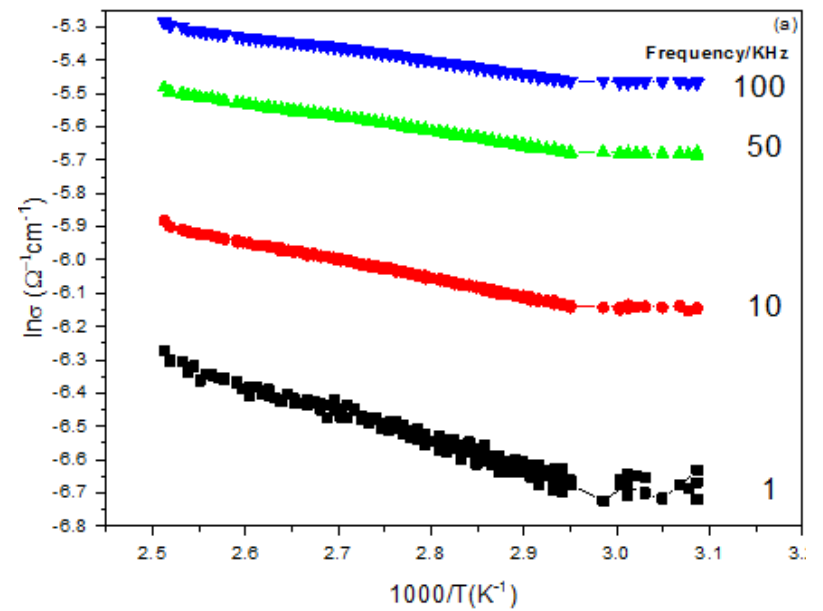

Figure 5. Ac conductivity as a function of temperature for: a) PEcbz-Ter at different frequencies and b) the PEcbz-Ter, PEcbz-Ant and PEcbz-Fur at $10 \mathrm{kHz}$.

\section{Conclusions}

The dielectric proprieties and ac conductivity of carbazole based materials PEcbz-Ter, PEcbz-Fur and PEcbz-Ant were studied as function of frequency (from $1 \mathrm{kHz}$ to $2 \mathrm{MHz}$ ) and temperature (from RT to $\left.120^{\circ} \mathrm{C}\right) . \varepsilon_{\mathrm{r}}^{\prime}$ was found to decrease with increasing frequency for all samples but increases with increasing temperature for PEcbz-Ter and PEcbzAnt. The values of $\tan \delta$ are found to be $0.05,0.14$ and 0.24 for the PEcbz-Fur, PEcbz-Ter and PEcbzAnt respectively at $11 \mathrm{kHz}$, which are below $1 \%$ and make them acceptable for many energy storage applications. The conductivity was found to increase very slightly with increase in temperature. According to the active energy values which obtained in this study, the hopping mechanism controls the behaviour conductivity of our materials.

\section{Acknowledgement}

We thank Prof. João Ramalho, Chemistry Department, Évora University for comments that greatly improved the manuscript.

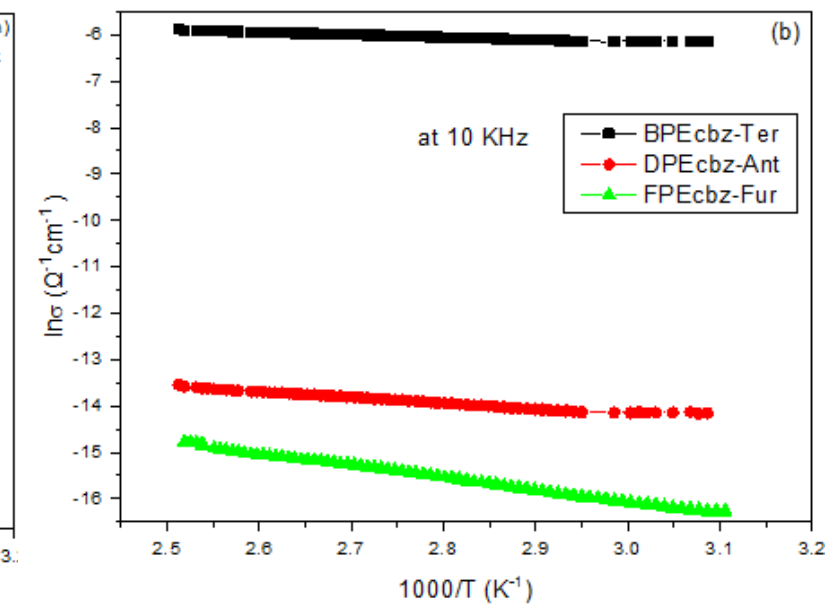

strong dependence on the frequency where it is increase with increasing frequency. The value of $\sigma_{\mathrm{ac}}$ versus temperature can be affected by the substitute in our copolymers, as shown in Figure $5 \mathrm{~b}$ at $10 \mathrm{kHz}$ where PEcbz-Ter has the higher value compared with PEcbz-Fur which has the lowest. In this study, the activation energies were determined from the slope of these curves at $10 \mathrm{kHz}(0.043 \mathrm{eV}, 0.099 \mathrm{eV}$ and $0.22 \mathrm{eV}$ for PEcbz-Ter, PEcbz-Ant and PEcbzFur respectively). The low values of active energy are in agreement with the hopping model in our copolymers. This behaviour is similar to conduction mechanism in plasma polymerized aniline thin films 19. The results of the PEcbz-Ter sample show favourable values more than PEcbz-Ant and PEcbzFur. This variation could be due to the different in composition according to the results shown in X-ray diffraction. 
photovoltaic properties for design of new AzoPyrrole photo-sensitizer materials as dyes in solar cells, J. Mater. Environ. Sci., 2017, 8, 2572-2578.

6- H. Bouaamlat, O. Ninis, M. Abarkan, M. Bouachrine, DFT and TD-DFT studies of alkyl-substituted oligopyrroles for optoelectronic devices, J. Mater. Environ. Sci., 2017, 8, 2651-2656.

7- A.T. Seyhan, Y. Göncü, O. Durukan, A. Akay, N. Ay, Silanization of boron nitride nanosheets (BNNSs) through microfluidization and their use for producing thermally conductive and electrically insulating polymer nanocomposites, J. Solid State Chem., 2017, 249, 98-107.

8- L. Valentini, I. Armentano, J. Biagiotti, A. Marigo, S. Santucci, J.M. Kenny, AC conductivity of conjugated polymer onto selfassembled aligned carbon nanotubes, Diam. Relat. Mater., 2004, 13, 250-255.

9- G. Garbay, L. Muccioli, E. Pavlopoulou, A. Hanifa, G. Hadziioannou, C. Brochon, E. Cloutet, Carbazole-based $\pi$-conjugated polyazomethines: Effects of catenation and comonomer insertion on optoelectronic features, Polymer., 2017, 119, 274-284.

10- R.A. Irgashev, N.A. Kazin, N.I. Makarova, I. V. Dorogan, V. V. Malov, A.R. Tameev, G.L. Rusinov, A. V. Metelitsa, V.I. Minkin, V.N. Charushin, Synthesis and properties of new $\pi$-conjugated imidazole/carbazole structures, Dyes Pigments., 2017, 141, 512-520.

11- A. Iraqi, I. Wataru, Preparation and Properties of 2, 7-Linked N -Alkyl-9 H -carbazole MainChain Polymers, 2004, 442-448.

12- J. Oriou, F. Ng, G. Hadziioannou, C. Brochon, E. Cloutet, Synthesis and structure-property relationship of carbazole-alt-benzothiadiazole copolymers, J. Polym. Sci. Part Polym. Chem., 2015, 53, 2059-2068.

13- M. Arbatti, X. Shan, Z. Cheng, Ceramicpolymer composites with high dielectric constant, Adv. Mater., 2007, 19, 1369-1372.
14- W. Wan, J. Luo, C. e. Huang, J. Yang, Y. Feng, W.X. Yuan, Y. Ouyang, D. Chen, T. Qiu, Calcium copper titanate/polyurethane composite films with high dielectric constant, low dielectric loss and super flexibility, Ceram. Int., 2018, 44, 5086-5092.

15- P. Brochu, Q. Pei, Advances in dielectric elastomers for actuators and artificial muscles, Macromol. Rapid Commun., 2010, 31, 10-36.

16- Y. Shi, L. Huang, A.K. Soh, G.J. Weng, S. Liu, S.A.T. Redfern, A scaling law for distinct electrocaloric cooling performance in lowdimensional organic, relaxor and antiferroelectrics, Sci. Rep., 2017, 7, 1-9.

17- H. Sadki, M.N. Bennani, K. Alimi, M. Bouachrine, Synthesis and Characterization of New Electroluminescent Material Based on Carbazole , and Terphenyle, 2014, 3 , 11600-11611.

18- Moha, Council for Innovative Research, J. Adv. Chem., 2014, 10, 2146-2161.

19- C.J. Mathai, S. Saravanan, M.R. Anantharaman, S. Venkitachalam, S. Jayalekshmi, Characterization of low dielectric constant polyaniline thin film synthesized by ac plasma polymerization technique, 2002, 35, 240-245.

20- O.E. Sherif, DFT calculations, electrical and thermal properties of unsubstituted triphenylformazan, Int. J. Electrochem. Sci., 2015, 10, 9751-9765.

21- G.M. Treich, S. Nasreen, A. Mannodi Kanakkithodi, R. Ma, M. Tefferi, J. Flynn, Y. Cao, R. Ramprasad, G.A. Sotzing, Optimization of Organotin Polymers for Dielectric Applications, ACS Appl. Mater. Interfaces., 2016, 8, 21270-21277.

22- G.E. Pike, ac Conductivity of Scandium Oxide and a New Hopping Model for Conductivity, Phys. Rev. B., 1972, 6, 1572-1580.

23- S.R. Elliott, Temperature dependence of a.c. conductivity of chalcogenide glasses, Philos. Mag. B., 1978, 37, 553-560. 